\title{
On the Occurrence of Large SSC Amplitudes in the Horizontal Intensity of the Geomagnetic Field in Middle Latitudes Around Local Midnight
}

\author{
B.J. SRIVAstava \\ National Geophysical Research Institute, Hyderabad, India \\ (Recived July 15. 1966)
}

\begin{abstract}
A study is made of the SSC amplitudes in $\mathrm{H}$ in relation to their local time of occurrence in the middle latitudes using data of 10 geomagnetic stations for the period 1953-60. It is found that (1) the greatest SSC amplitudes in $H$ are recorded (in the summer months) around the local midnight ( 1 to 3 hours before or 1 to 3 hours afterthe local midnight), and (2) the minimum in SSC amplitudes in $H$ occurs (in the winter months) during $18^{h}-21^{h}$ L.T. at the African stations and during $4^{h}-10^{h}$ L.T. (generally around $7^{h}$ L.T.) at the other stations.

The midnight maximum amplitude and the morning minimum amplitude are explained in terms of the ionospheric screening effect and precipitation of electrons into the auroral zone ionosphere on the night side of the earth from the outer Van Allen Belt at the time of the SSC.
\end{abstract}

\section{Introduction}

In a discussion of the three major SC-type geomagnetic storms recorded at the Indian magnetic observatories on 11, 15 and 17 July 1959, it was pointed out by Srivastava (1961) that at Alibag the amplitudes of those SSC's in $H$ were well above the average value $(30 \gamma)$ and that the SSC's on 11 and 17 July occurred during the night time. Furthermore, in a table were presented the large SSC's $(\Delta H>75 \gamma)$ recorded at Alibag during the period $1921-$ 1958. There were 14 such SSC's of which 7 occurred during night hours and 7 during day hours. But those occurring in night hours were of comparatively greater amplitude than those recorded in day time. It was thus inferred that even though occurrence of a large SSC $(\Delta H>75 \gamma)$ at Alibag in night hours and in day hours is equally probable, those occurring around local midnight are comparatively greater in amplitude. Besides this, the observation of Maeda and Yamamoto (1960) that the effect of the equatorial electrojet does not extend beyond $20^{\circ}$ of Dip (Geomagnetic Inclination) on either side of the magnetic equator, was also verified in the case of Alibag which with a mean Dip of $24^{\circ} 38^{\prime}$ for 1958 was found to be just outside the influence of the equatorial electrojet.

Ferraro $(1951,1954)$ examined the SSC-amplitudes in relation to their local time of occurrence at the five non-equatorial stations of Cheltenham, Tucson, San Juan, Honolulu and Watheroo for the period 1926-46 and found that unlike Huancayo, these stations did not show a day-time augmentation of the mean hourly values of the main SSC impulse. In the 
single graph presented by Ferraro for the five stations jointly, it is seen that the maximum SSC- amplitude occurs around $22^{h}$ Local Time (night time). A secondary maximum, lower than the main maximum, appears around $13^{h}$ L.T. (day time). The minimum amplitude occurs around $8^{h}$ L.T. (morning hours). This occurrence of minimum SSC-amplitude around $8^{h}$ L.T. was explained by Ferraro as due to the fact that over the day-lit hemisphere the higher conductivity in the ionosphere tends to shield the stations below from the external magnetic field produced by the interaction of the solar corpuscular stream with the geomagnetic field several earth radii away.

Kazmi (1963) has presented a graph of SC and SI amplitude in $H$ against local time in respect of the magnetic station at Quetta for the period 1953-1962, in which the maximum amplitude occurs around $3^{h}$ L.T. (night time). Secondary maxima appear around $13^{h}$ L.T. (day time) and $23^{h}$ L.T. (night time). The minimum amplitude occurs around $7^{h}$ L.T. (morning hours).

Nishida (1964) has made a theoretical study of the ionospheric screening effect and storm sudden commencement by solving Maxwell's electromagnetic equations in the neutral atmosphere, in the ionosphere, and in the magnetosphere. He derives a relation between the incident and the observed field. It seems that the main change (SSC and main impulse of SSC*) is related to an incident mode which is affected but little by the ionosphere and is explicable by the compression of the magnetosphere as generally considered (Chapman and Ferraro, 1931 ; Dessler and Parker, 1959). The preceding reverse change (preceding reverse impulse of SSC*), however, corresponds to an incident mode which is subject to ionospheric modification in terms of rotation of the vector. The preceding reverse impulse results from the deformation of the magnetosphere due to the viscous force exerted by the intensified solar corpuscular stream which generates in the magnetosphere an eastward flow in the afternoon side of the earth and a westward flow in the morning side. He, however, does not precisely indicate the effect of the ionosphere on the magnitude of SSC's.

Table I

\begin{tabular}{l|c|c|c|r}
\hline \multirow{2}{*}{ Station } & \multicolumn{3}{|c}{ Geographic Coordinates Geomagnetic Coordinates. } \\
\cline { 2 - 5 } & Latitude & Longitude & Latitude & Longitude \\
\hline Honolulu & $21^{\circ} 18^{\prime} \mathrm{N}$ & $158^{\circ} 06^{\prime} \mathrm{W}$ & $21^{\circ} .0$ & $266^{\circ} .4$ \\
Tucson & $32^{\circ} 14^{\prime} \mathrm{N}$ & $110^{\circ} 57^{\prime} \mathrm{W}$ & $40^{\circ} .4$ & $312^{\circ} .1$ \\
San Juan & $18^{\circ} 23^{\prime} \mathrm{N}$ & $66^{\circ} 07^{\prime} \mathrm{W}$ & $29^{\circ} .9$ & $3^{\circ} .2$ \\
Vassouras & $22^{\circ} 24^{\prime} \mathrm{S}$ & $43^{\circ} 40^{\prime} \mathrm{W}$ & $-11^{\circ} .9$ & $23^{\circ} .8$ \\
Leopoldville/ & $04^{\circ} 22^{\prime} \mathrm{S}$ & $15^{\circ} 15^{\prime} \mathrm{E}$ & $-3^{\circ} .1$ & $83^{\circ} .5$ \\
Binza & $34^{\circ} 25^{\prime} \mathrm{S}$ & $19^{\circ} 13^{\prime} \mathrm{E}$ & $-33^{\circ} .3$ & $80^{\circ} .3$ \\
Hermanus & $11^{\circ} 38^{\prime} \mathrm{S}$ & $27^{\circ} 25^{\prime} \mathrm{E}$ & $-12^{\circ} .7$ & $94^{\circ} .1$ \\
Elizabeth- & $18^{\circ} 38^{\prime} \mathrm{N}$ & $72^{\circ} 52^{\prime} \mathrm{E}$ & $9^{\circ} .5$ & $143^{\circ} .6$ \\
ville/Karavia & $30^{\circ} 19^{\prime} \mathrm{S}$ & $115^{\circ} 53^{\prime} \mathrm{E}$ & -41.7 & $185^{\circ} .8$ \\
Alibag & $31^{\circ} 56^{\prime} \mathrm{S}$ & $115^{\circ} 57^{\prime} \mathrm{E}$ & -43.4 & $185^{\circ} .8$ \\
Watheroo/ & $13^{\circ} 48^{\prime} \mathrm{S}$ & $171^{\circ} 46^{\prime} \mathrm{W}$ & $-16^{\circ} .0$ & $260^{\circ} .2$ \\
Gnangara & &
\end{tabular}




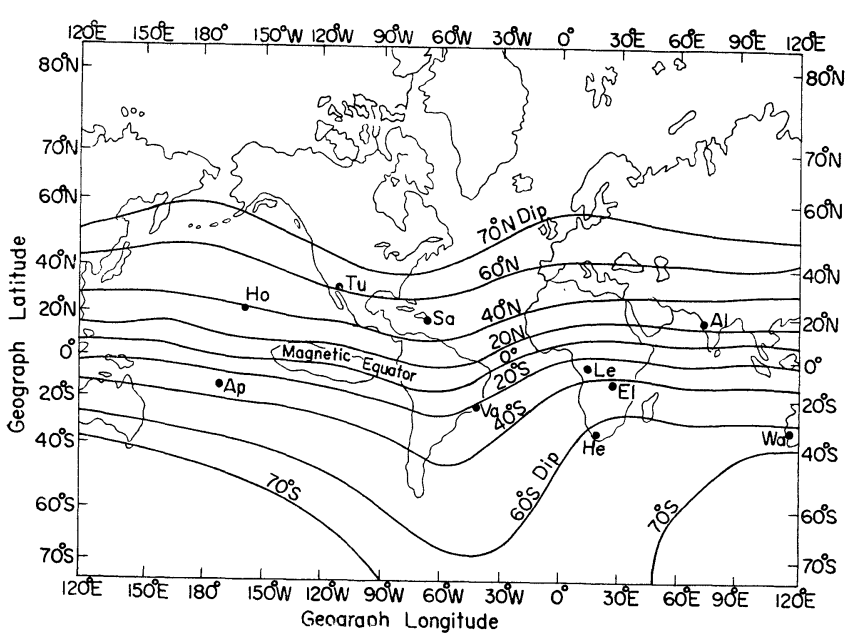

Fig. 1. Distribution of the 10 geomagnetic sta-tions on the world map along with isoclinics.

The present study was undertaken with a view to examining the night-time occurrence of maximum amplitude of SCC in the horizontal intensity $(H)$ of the geomagnetic field in the middle latitudes. For this purpose, 10 existing geomagnetic stations were selected round the world in the middle latitudes in the northern as well as southern hemisphere. These stations are outside the $20^{\circ}$ Dip line on either side of the magnetic equator and also outside the polar and auroral zones, so that these stations are outside the influence of the equatorial and auroral electrojets. The following table gives the stations and their geographic and geomagnetic coordinates (Table I). Fig. 1 shows the distribution of these geomagnetic stations on the world map.

\section{The Data}

The time of occurrence and the amplitude of SSC's in $H$ in gammas as published in the Journal of Geophysical Research (Washington) for the 8-year period 1953-1960, in respect of the 10 stations mentioned above have been used in the present investigation. The sunspot minimum, with which the 19th solar cycle began, occurred in June 1954, and the sunspot maximum in October 1957. The 8 years' data thus chosen spread over half a sunspot cycle and $11 / 2$ years before the minimum and 3 years after the maximum sunspot year.

The time of occurrence of the SSC's (U.T.) were converted into local time (L.T.) for each station. Then the data of each station were grouped according to the three seasons $D, E \& J$; namely, $D$ for the December Solstice (January, February, November and December), $E$ for the Equinoctial season (March, April, September and October) and $J$ for the June Solstice (May, June, July and August). For each season the hourly values of the main SSC impulse were then formed by averaging the amplitudes of all positive SSC's occurring over the one-hour intervals centred at full hours L.T. Those occurring at half hours were considered for both the full hours during which the half-hour occurred. Thus for each station for each of the three seasons the mean diurnal variation of positive SSC main impulse amplitudes was formed. The mean diurnal variation of hourly amplitudes for each station for the 8-year period was also formed by taking the mean of the corresponding hourly values for the three seasons. 


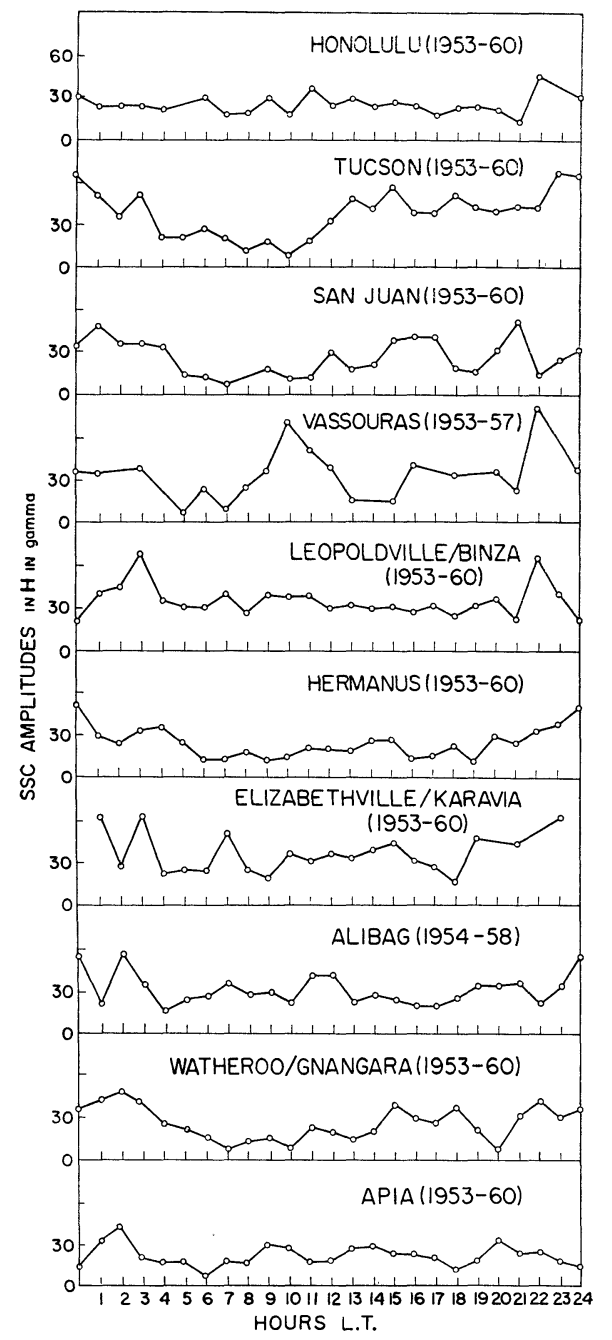

Fig. 2. Diural variation of ssc amplitudes in the horizontal geomagnetic intensity $(H)$ at the 10 stations.

\section{Analysis and Discussion}

The mean graphs of SSC amplitude against local time of its occurrence in respect of each ofthe 10 stations are given in Fig. 2.

Following are the station-wise noteworthy features of the graphs:-

1. Honolulu. Based on scanty data. Main $\max$ at $22^{h}$ (L.T.), secondary max. at $11^{h}$ (L.T.) Main $\min$ at $21^{h}$ (L.T.), Secondary $\min$ at $7^{h}$ (L.T.)-difference of $4 \gamma$ in the two minima. Ratio of the max. amp. to day-time min. amp. is $45 /$ 17 i.e. 2.6 .

2. Tucson. Main max. at $23^{h}$ (L.T.), Secondary $\max$. at $15^{h}$ (L.T.). Min. at $10^{h}$ (L.T.). Ratio of max. amp. to min. amp. is $66 / 8$. i.e. 8.3. Greatest amp. occurs in Summer Season.

3. San Juan. Main max. at $21^{h}$ (L.T.), Secondary max. at $16^{h}$ (L.T.). Main min. at $7^{h}$ (L.T.), Secondary min. at $19^{h}$ (L.T.). Ratio of max. amp. to min. amp. is $52 / 7$ i.e. 7.4 .

4. Vassouras. Based on scanty data. Main max. at $22^{h}$ (L.T.), prominent secondary max. at $10^{h}$ (L.T.). Main min. at $5^{h}$ and $7^{h}$, secondary min. around $14^{h}$ (L.T.). Ratio of max. amp. to min. amp. $\left(7^{h}\right)$ is $80 / 9$ i.e. 8.9 . The secondary max. at $10^{h}$ L.T. is remarkable and is perhaps indicative of Vassouras being slightly affected by the equatorial electrojet (Dip of Vassouras is $-23^{\circ}$ ).

5. Leopoldville/Binza. Main max. at $3^{h}$ (L.T.), Secondary max. at $22^{h}$ (L.T.). Main min. amp. at $21^{h}$ (L.T.), Secondary min. at $18^{h}$ and $8^{h}$ (L.T.). Ratio of $\max$. to $\min \left(8^{h}\right)$ is $68 / 26$ i.e. 2.6. Greatest amp. occurs in Summer months.

6. Hermanus. Main max. at $0^{h}$ (L.T.), Secondary max. $4^{h}$ L.T. and $15^{h}$ (L.T.). Main min. at $19^{h}$ (L.T.), Secondary min. at $9^{h}$ and $16^{h}$ (L.T.). Ratio of max. to day min. $\left(9^{h}\right)$ amp. is $52 / 12$ i.e. 4.3. Greatest amplitude occurs in Summer months.

7. Elizabethville/Karavia. Based on scanty data. Main max. around $0^{h}$ (L.T.), secondary max. at $7^{h}$ (L.T.), and $15^{h}$ (L.T.). Main min at $18^{h}$ (L.T.), secondary min. at $9^{h}$ (L.T.). Ratio of max. amp. to day time min. amp. is $63 / 19$ i.e. 3.3 .

8. Alibag. Main max. amp. at $2^{h}$ (L.T.), secondary max. around $0^{h}$ and $1130^{h}$. Main min. at $4^{h}$ (L.T.), secondary min. at 1630 (L.T.). Ratio of max. to min. amp. is $57 / 18$ i.e. 3.2 . 
Greatest amp. occurs in summer momths, especially in the month of July.

9. Watheroo/Gnangara. Main max. amp. at $2^{h}$ (L.T.), secondary max. at $22^{h}$ (L.T.) and $15^{h}$ (L.T.). Main min. at $7^{h}$ (L.T.), secondary min. at $20^{h}$ (L.T.) Ratio of max. amp. to day time min. amp. is $48 / 6$ i.e. 8.0 .

10. Apia. Main maximum amplitude occurs at $2^{h}$ (L.T.), a secondary max. at $20^{h}$ (L.T.). Minimum amplitude occurs at $6^{h}$ (L.T.), a secondary minimum at $18^{n}$ (L.T.). The ratio of maximum amp. to day time minimum amp. is $44 / 7$ i.e. 6.3 . Geatest amplitude is recorded in Summer Solstice around $2^{h}$ (L.T.).

To this may be added the chief features of the mean diurnal variation of SSC and SI amplitudes obtained by Kazmi (1963) in respect of Quetta based on 10 years' data from 1953 to 1962, which are as already stated in the Introduction: The main max. amp. occurs around $3^{h}$ (L.T.), secondary max. around $13^{h}$ and $23^{h}$ (L.T.). Min. amp. occurs around $7^{h}$ (L.T.). Ratio of max. amp. to min. amp. is 40/16 i.e. 2.5. Greatest amp. occurs in July and August (Summer months).

It will be at once noticed that the main maximum SSC amplitude in $H$ occurs at stations west of Leopoldville right upto Honolulu 1 to 3 hours before the local midnight, and at stations east of Leopoldville right upto Watheroo/Gnangara 1 to 3 hours after the local midnight. At Hermanus and Elizabethville, it occurs just around local midnight.

It has to be emphasised that the above results are based on only 8 years' data, and as such the seasonal features of the graphs cannot be asserted statistically. However, the main features of the mean SSC amplitude diurnal variation graphs (Fig. 2) are indisputable.

\section{Conclusion}

The following conclusions can be drawn from the above study:-

(i) In the middle latitudes (outside the influence of the electrojets) greatest SSC amplitudes in $H$ are recorded in the Summer months around the local midnight at the African stations; 1 to 3 hours before local midnight at the American stations; and 1 to 3 hours after the local midnight at the Indian and Australian stations.

(ii) The minimum in SSC amplitudes in $H$ occurs in the winter months during $18^{h}-21^{h}$ (L.T.) at the African stations; during $4^{h} / 10^{h}$ generally around $7^{h}$ (L.T.) at the Indian, Australian and American stations.

It would be quite interesting to correlate the above two conclusions regarding SSC amplitudes with the ionospheric conductivity over the respective areas at those hours. Kamiyama and Sato (1957) have given curves for longitudinal distribution of integrated conductivities (Cowling, Pedersen and Hall, separately) of the ionosphere ( $E$ and $F$ regions) for different latitudes. In all these curves, the minimum conductivity occurs around $6^{h}$ (L.T.), and immediately after that there is an abrupt rise till about $7^{h}$ (L.T.) (which might be called the sunrise effect), after which the rise in conductivity to the max. value at $12^{h}$ L.T. is rather small and gradual. It is for this reason, perhaps, that the max. screening effect of ionosphere on the SSC amplitudes is observed around $7^{h} \mathrm{~L}$.T. The minimum screening effect of the ionosphere and consequently the max. SSC amplitudes should be recorded around $6^{h}$ L.T., 
according to the same conductivity curves. But the maximum amplitudes are actually recorded around the local midnight as seen from Fig. 2. This might be due to additional ionospheric currents caused by precipitation of electrons into the auroral zone ionosphere on the dark side of the earth from the outer Van Allen Belt at the time of the SSC (Barcus, 1965) in conjunction with the already precipitated protons from the solar wind. The current systems are similar to those for SD or geomagnetic bays which give an additional amplitude to the SSC's occurring around local midnight in middle latitudes. These currents do not exist around $6^{h}$ L.T.. Hence the SSC amplitudes around local midnight are greater than the amplitudes around $6^{h}$ L.T., although the ionospheric screening is lesser around $6^{h}$ L.T. than around midnight.

\section{Acknowledgements}

I am thankful to Dr. P.V. Sanker Narayan of the National Geophysical Research Institute for helpful discussions and suggestions in the course of preparation of this paper. $\mathrm{My}$ grateful thanks are also due to the Director, National Geophysical Research Institute, for his kind permission to publish this paper.

\section{References}

Barcus, J.R., Evidence suggesting dumping of semitrapped electrons on the night side of the earth, J. Geophys. Res., 70, 1237-1239, 1965.

Chapman, S. and V.C.A. Feraro, Terr. Mag. Atmos. Electr. 36, 77-97, 171-186, 1931.

Dessler, A.J. and E.N. Parker, Hydromagnetic theory of geomagnetic storms, J. Geophys. Res. 64, 22392252, 1959.

Ferraro, V.C.A. and H.W. Unthank, Sudden Commencement and Sudden Impulses in Geomagnetism : their diuranl variation in amplitude, Geofis. Pura e Applicata, 20, 27-30, 1951,

Ferraro, V.C.A., Some remarks on recent notes by Drs. Sugiura and Vestine. J. Geophys. Res. 59, 309$311,1954$.

Kamiyama, H. and T. Sato: The distribution of the electrical conductivity in the ionosphere and its variations ; Sci. Rep. Tohoku Univ., Fifth series (Geophysics), 9, 55-79, 1957.

Kazmi, S.A.A., Diurnal variation in the amplitude and frequency of magnetic sudden commencement and sudden impulses at Quetta, J. Geomag. \& Geoelect. (Japan), 15, 109-115, 1963.

Nishida, A., Ionospheric screening effect and storm sudden commencement, J. Geophys. Res., 69, 1861$1874,1964$.

Shirgaokar, A.J. and B.J. Srivastava, Geomagnetic Storms in July 1959, Proc. IGY Symposium, Vol. II, Feb. 13-16 1961, New Delhi, 74-77, 1961, 